Journal of Prosthodontic Research (revised version) Case report

A nine-year clinical case study of a resin-bonded fixed partial denture seated on the maxillary anterior teeth

Abbreviated title: A nine-year clinical case study of a resin-bonded fixed partial denture

Keywords: abutment tooth, edge fracture, noble metal, resin-bonded fixed partial denture, thiol.

The number of pages in the text; 11, number of tables; 0 ,

number of figures; 4, the quantity of reprints desired; 30 


\section{Clinical significance}

The use of vinyl-thiol primer and tri-n-butylborane initiated adhesive resin is a clinically reliable bonding system for seating resin-bonded fixed partial denture (RBFPD) made from a silver-palladium-copper-gold alloy. The RBFPD, based on a reliable bonding system, can

clinically function for a long time, even if the vertical overlap of the abutment teeth is excessive. 


\section{Abstract}

Patient: This report describes the longevity of a resin-bonded fixed partial denture (RBFPD). This denture was seated on the maxillary anterior teeth with minimal tooth preparation. The RBFPD was cast from a silver-palladium alloy (Castwell M.C. 12), and the pontic was veneered with an indirect composite material (Estenia). The retainers were primed with a metal conditioner (V-Primer) and seated with a tri-n-butylborane initiated adhesive resin (Super-Bond C\&B). After an observation period of eight years, a fracture occurred in the incisal edge of the central incisor abutment. The fractured area was restored with light-polymerizing composite resin and the anterior guidance was re-adjusted.

Discussion: RBFPD abutment teeth with deep vertical overlap should be carefully prepared to avoid abutment tooth fracture.

Conclusion: The clinical performance of the RBFPD made from a silver-palladium-copper-gold alloy was sufficient when seated with tri-n-butylborane initiated adhesive resin after surface modification using vinyl-thiol primer. 


\section{Introduction}

Resin-bonded fixed partial dentures (RBFPDs) are now widely accepted in clinical practice together with the concept of minimal intervention. Numerous studies reporting the clinical performance of RBFPDs have been published, and their usefulness has already been established by much evidence [1-9].

The reason for failure of RBFPDs had long been "debonding of the framework from the abutment teeth”. Nevertheless, the development and/or improvement of the adhesive system successfully changed the clinical and technical procedures of RBFPD treatment. The adhesive system involves the mechanical and chemical bonding system between metal frameworks and acrylic luting agents, and between acrylic luting agents and tooth structure. The longevity of RBFPD is now comparatively higher than before by use of the appropriate metal alloy, acrylic luting agent, and metal conditioner.

Regarding current RBFPDs, problems other than debonding are now indicated. A fracture of the tooth structure of the abutment teeth is one of the troubles that can happen to the RBFPD treatment regardless of the reliability of the bonding system. This clinical report describes the nine-year clinical performance of an RBFPD seated on the maxillary anterior teeth to restore esthetics and oral function. This report also addresses the treatment for an edge fracture of the central incisor, an abutment tooth, which occurred eight years after insertion. 


\section{Outline of the case}

A 62-year-old male patient presented for periodontal rehabilitation (Fig. 1). As a result of intraoral and radiographic examinations, he was recommended the extraction of the maxillary left central incisor for endodontic and periodontal reasons. Several treatment options after tooth extraction were proposed in advance: 1) single-tooth implant, 2) RBFPD with minimal tooth reduction, 3) direct-bonding of an artificial pontic, and 4) a removable partial denture similar to a removable retainer. Among the proposed techniques, the patient preferred the second procedure. Further details of the treatment were explained and the patient's consent to the RBFPD was obtained.

After tooth extraction, occlusion was examined intraorally with pieces of articulating paper and extraorally using a diagnostic cast. After the examination of maxillomandibular relationship, the areas and the amount of tooth reduction were determined. The bonding area of the retainers was extended as much as possible within the lingual surfaces except the edge area. Past literature has indicated that reliable bonding was obtained without additional grooves or holes [10]. Thus, we elected to minimize tooth preparation and not to use these retentive features.

A combined impression of the entire maxilla was made with silicone elastomeric materials (Exafine Regular and Injection, GC Corp., Tokyo, Japan) using a custom tray, and centric relation was registered using a vinyl polysiloxane impression material (Exabite II NDS; GC America Inc., Chicago, Ill, USA). The impression was poured with a die stone, 
and a working cast was prepared. The stone cast was then mounted on an articulator with an opposing cast. The pattern of the retainer and pontic was fabricated with an inlay wax material (Blue Inlay Wax, GC Corp.) and invested with a cristobalite investing material (Cristobalite Micro, GC Corp.). The metal framework that consisted of two retainers and a pontic was cast from a silver-palladium-copper-gold alloy (Castwell M.C. 12, GC Corp.). The alloy was selected because of its higher bonding performance compared to other dental metal alloys [11]. The pontic was of the ridge-lap type. After heat treatment of the alloy, the buccal surface of the pontic was veneered with an indirect composite material (Estenia, Kuraray Medical Inc., Tokyo, Japan).

On the next visit of the patient, the completed RBFPD was tried in. The inner surfaces of the retainer were air-abraded with 50-70 $\mu \mathrm{m}$ grain sized aluminum oxide (Hi-Aluminas, Shofu Inc., Kyoto, Japan) using a particle abrader (Micro Blaster MB102, Comco Inc., Burbank, CA, USA) and conditioned with a single liquid vinyl-thiol primer designed for noble metals (V-Primer, Sun Medical Co. Ltd., Moriyama, Japan) [12]. The abutment lingual enamel surfaces were etched with phosphoric acid (Red Activator, Sun Medical Co. Ltd.), washed with water, and air-dried. The RBFPD was then seated with a tri-n-butylborane (TBB) initiated adhesive resin (Super-Bond C\&B Opaque, Sun Medical Co. Ltd.) applied using a brush-dip technique. Fig. 2 shows the intraoral views immediately after seating the RBFPD. The patient thereafter entered a maintenance program of three month intervals. 
After eight years from insertion, the patient complained of a fracture of the edge of the maxillary central incisor (Fig. 3). He could not specify when and how the fracture occurred. The patient did not feel pain and prosthesis did not lose retention, although recurrent caries was observed. He was satisfied with the RBFPD aesthetically, although the luster of the veneering material was obviously deteriorated.

The fractured portion was filled with a light-polymerizing restorative composite resin (Palfique Estelite, Tokuyama Corp., Tokyo, Japan). After the filling, the anterior guidance was re-checked and re-adjusted using occlusal equilibration, and the composite was

polished (Fig. 4). The patient was then re-instructed how to maintain oral hygiene. The RBFPD has been functioning satisfactorily for about ten years without debonding.

\section{Discussion}

The effectiveness of the RBFPD system of the silver-palladium-copper-gold alloy, vinyl-thiol primer, and TBB initiated acrylic luting agent is already well known [12,13]. The experimental in vitro results also support the reliability of the RBFPD system [14]. Some base metal alloys have been often used for RBFPD, and many mechanical and chemical treatment methods for base metal bonding surface have also been introduced and clinically evaluated [15-23]. However, the rate for debonding of the silver-palladium-copper-gold RBFPD framework applied with vinyl-thiol primer has not been reported to be higher than previous RBFPD systems [13]. 
The development of dental adhesive systems has enabled the retention of sufficient intact tooth structure of the abutment teeth. Nevertheless, the intact area of the abutment tooth is, of course, exposed to occlusal force, and the RBFPD design based on minimal intervention cannot prevent fracture of the abutment teeth.

In the current case, the metal framework was undamaged, probably due to strengthening of the mechanical properties of the metal alloy by heat treatment performed prior to the veneering procedure. The composite edge of the opposite incisor (pontic) was also unbroken, since a highly-filled (92 wt\%) composite was selected for veneering. As a result, the fracture occurred only in the abutment tooth because of the insufficient occlusal adjustment at insertion and the expansion of the caries that already existed when seating the RBFPD (Fig. 2).

If an intact tooth fragment is present, the incisal edge reattachment procedure presents a conservative, simple and aesthetic treatment. However, the patient could not specify when and how the fracture occurred, and lost the fragment. Another way to treat the fracture is, of course, to restore using composite materials. In fact, clinical trials and long-term follow-up have reported the reattachment using dentine bonding agents or adhesive luting systems may achieve functional and aesthetic success for up to 7 years [24]. Nevertheless, the vertical overlap in this case was excessively deep and the risk of edge fracture by the protrusive movement of the mandible was anticipated by the intraoral and extraoral examination. Only the occlusal equilibration was therefore performed as to incisal 
edge to avoid debonding or fracture of the composite filling material, although the fractured portion except the incisal edge was filled with a restorative composite resin as usual.

The bonding (prepared) areas in the lingual walls of the abutment teeth did not extend to the edge (Fig. 2B) in this case, considering the susceptibility of the edge area due to thin tooth substance. If the bonding area had extended to the edge, the fracture of the edge could have been avoided. However, the color of the abutment teeth would have been artificially whitened by the opaque-colored luting agent used for seating the RBFPD. From this viewpoint, it seems that the original design was correct. Yet, it is not to be denied that the occlusal equilibration should be performed before tooth preparation.

Fortunately, the fracture of the abutment tooth in this case was not serious. Needless to say, it could have been because the bond strength between the abutment tooth and RBFPD was satisfactorily high, and the tooth substance was protected by the RBFPD itself. The fracture in this case is so-called "cohesive failure inside the substrate material without interface separation”. Consequently, this failure mode substantiated the efficiency of the RBFPD bonding system adopted.

The use of vinyl-thiol primer and TBB initiated adhesive resin is a clinically reliable bonding system for seating RBFPD made from a silver-palladium-copper-gold alloy. However, it is still important to carefully prepare the abutment teeth and adjust the occlusal contact, since the RBFPD structure cannot prevent recurrent caries and fracture of abutment teeth substances. 


\section{Conclusions}

The clinical performance of the RBFPD made from a silver-palladium-copper-gold alloy was sufficient when seated with tri-n-butylborane initiated adhesive resin after surface modification using vinyl-thiol primer. This reliable bonding system can contribute to realization of minimal intervention, although the abutment tooth should be carefully prepared to avoid abutment tooth fracture. The adjustments of the occlusal contacts of the abutment tooth and RBFPD metal framework are important, especially for a case with excessive vertical overlap. 


\section{REFERENCES}

1. Hansson O, Moberg LE. Clinical evaluation of resin-bonded prostheses. Int J Prosthodont 1992;5:533-41.

2. Isidor F, Stokholm R. Resin-bonded prostheses for posterior teeth. J Prosthet Dent 1992;68:239-43.

3. Hansson O, Bergstöm B. A longitudinal study of resin-bonded prostheses. J Prosthet Dent 1996;76:132-9.

4. Stokholm R, Isidor F. Resin-bonded inlay retainer prostheses for posterior teeth. A 5-year clinical study. Int J Prosthodont1996;9:161-6.

5. De Kanter RJ, Creugers NH, Verzijden CW, Van't Hof MA. A five-year multi-practice clinical study on posterior resin-bonded bridges. J Dent Res 1998;77:609-14.

6. Zalkind M, Ever-Hadani P, Hochman N. Resin-bonded fixed partial denture retention: a retrospective 13-year follow-up. J Oral Rehabil 2003;30:971-7.

7. Ketabi AR, Kaus T, Herdach F, Groten M, Axmann-Krcmar D, Pröbster L, et al. Thirteen-year follow-up study of resin-bonded fixed partial dentures. Quint Int 2004;35:407-10.

8. Garnett MJ, Wassell RW, Jepson NJ, Nohl FS. Survival of resin-bonded bridgework provided for post-orthodontic hypodontia patients with missing maxillary lateral incisors. Br Dent J 2006;201:527-34. 
9. Andenino G, Giannella G, Morello GM, Ceccarelli M, Carossa S, Bassi F. Resin-bonded fixed partial dentures: ten-year follow-up. Int $\mathrm{J}$ Prosthodont 2006;19:22-3.

10. Tanaka T, Atsuta M, Nakabayashi N, Masuhara E. Surface treatment of gold alloys for adhesion. J Prosthet Dent 1988;60:271-9.

11. Matsumura H, Kamada K, Tanoue N, Atsuta M. Effect of thione primers on bonding of noble metal alloys with an adhesive resin. J Dent 2000;28:287-93.

12. Hikage S, Hirose Y, Sawada N, Endo K, Ohno H. Clinical longevity of resin-bonded bridges bonded using a vinyl-thiol primer. J Oral Rehabil 2003;30:1022-9.

13. Tanoue N, Ide T, Kawasaki K, Nagano K. Tanaka T. Survival of resin-bonded fixed partial dentures made from a silver-palladium-copper-gold alloy. Int Chin J Dent 2006;6:53-9.

14. Matsumura H, Taira Y, Atsuta M. Adhesive bonding of noble metal alloys with a triazine dithiol derivative primer and an adhesive resin. J Oral Rehabil 1999;26:877-82.

15. Hansson O, Moberg LE. Clinical evaluation of resin-bonded prostheses. Int J Prosthodont 1992;5:533-41.

16. Isidor F, Stokholm R. Resin-bonded prostheses for posterior teeth. J Prosthet Dent 1992;68:239-43.

17. Hansson O, Bergstöm B. A longitudinal study of resin-bonded prostheses. J Prosthet Dent 1996;76:132-9. 
18. Stokholm R, Isidor F. Resin-bonded inlay retainer prostheses for posterior teeth. A 5-year clinical study. Int J Prosthodont 1996;9:161-6.

19. De Kanter RJ, Creugers NH, Verzijden CW, Van't Hof MA. A five-year multi-practice clinical study on posterior resin-bonded bridges. J Dent Res 1998;77:609-14.

20. Zalkind M, Ever-Hadani P, Hochman N. Resin-bonded fixed partial denture retention: a retrospective 13-year follow-up. J Oral Rehabil 2003;30:971-7.

21. Ketabi AR, Kaus T, Herdach F, Groten M, Axmann-Krcmar D, Pröbster L, Weber H. Thirteen-year follow-up study of resin-bonded fixed partial dentures. Quintessence Int 2004;35:407-10.

22. Garnett MJ, Wassell RW, Jepson NJ, Nohl FS. Survival of resin-bonded bridgework provided for post-orthodontic hypodontia patients with missing maxillary lateral incisors. Br Dent J 2006;201:527-34.

23. Andenino G, Giannella G, Morello GM, Ceccarelli M, Carossa S, Bassi F. Resin-bonded fixed partial dentures: ten-year follow-up. Int J Prosthodont 2006;19:22-3.

24. Murchison DF, Burke FJ, Worthingtom RB. Incisal edge reattachment: indications for use and clinical technique. Br Dent J 1999;186:614-9. 
Figure legends

Fig. 1. Intraoral frontal view (A) and radiograph (B) before extraction.

Fig. 2. Intraoral frontal (A) and lingual (B) views of the RBFPD immediately after seating.

Fig. 3. Intraoral frontal (A) and lingual (B) views of the RBFPD eight years after seating. Incisor edge fracture of the abutment tooth could be observed.

Fig. 4. Intraoral frontal (A) and lingual (B) views of RBFPD nine years after seating. The incisor edge fracture filled with restorative composite was unattractive due to occlusal equilibration. 
Fig. 1

(A)

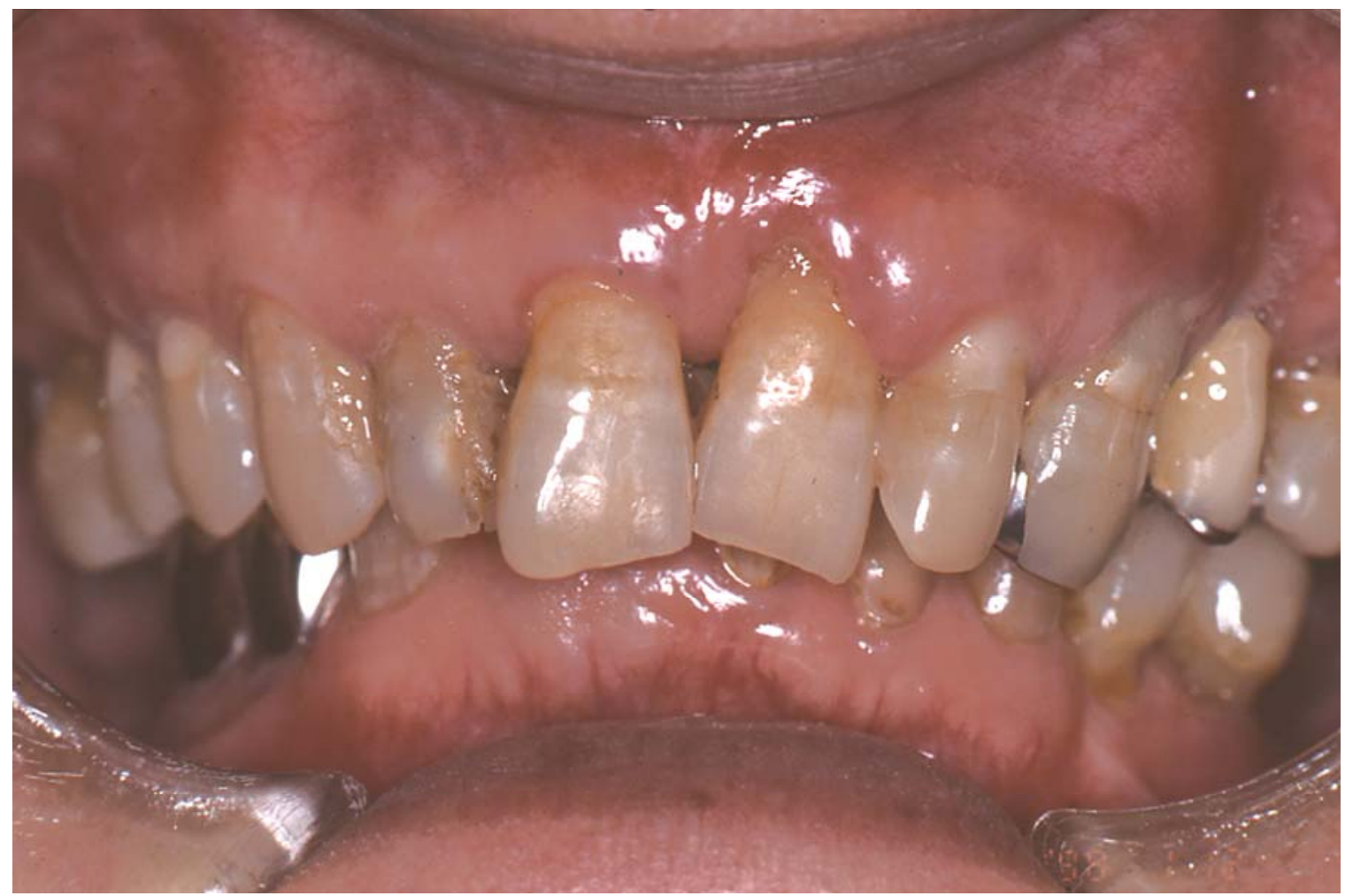


Fig. 1

(B)

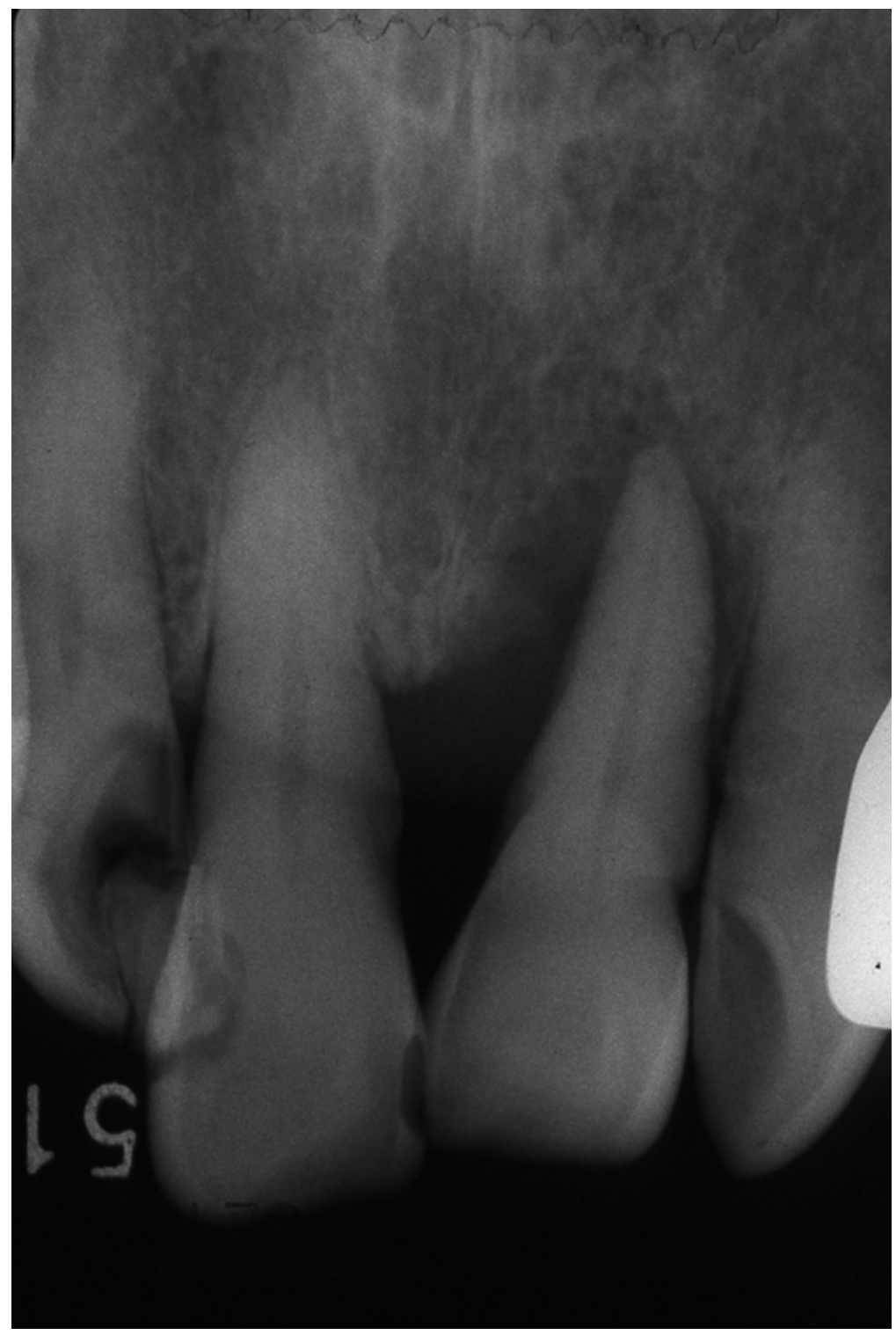


Fig. 2

(A)

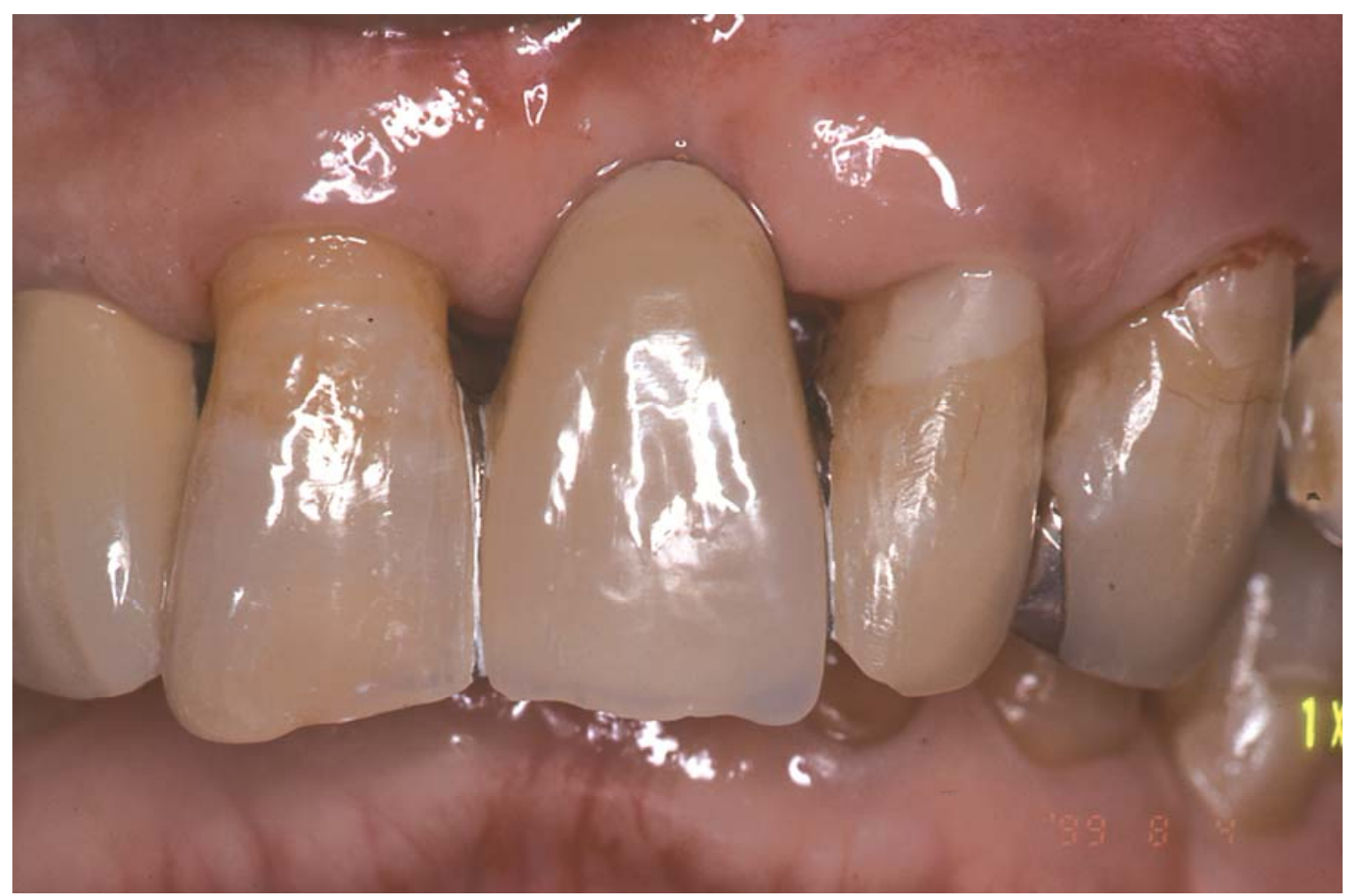


Fig. 2

(B)

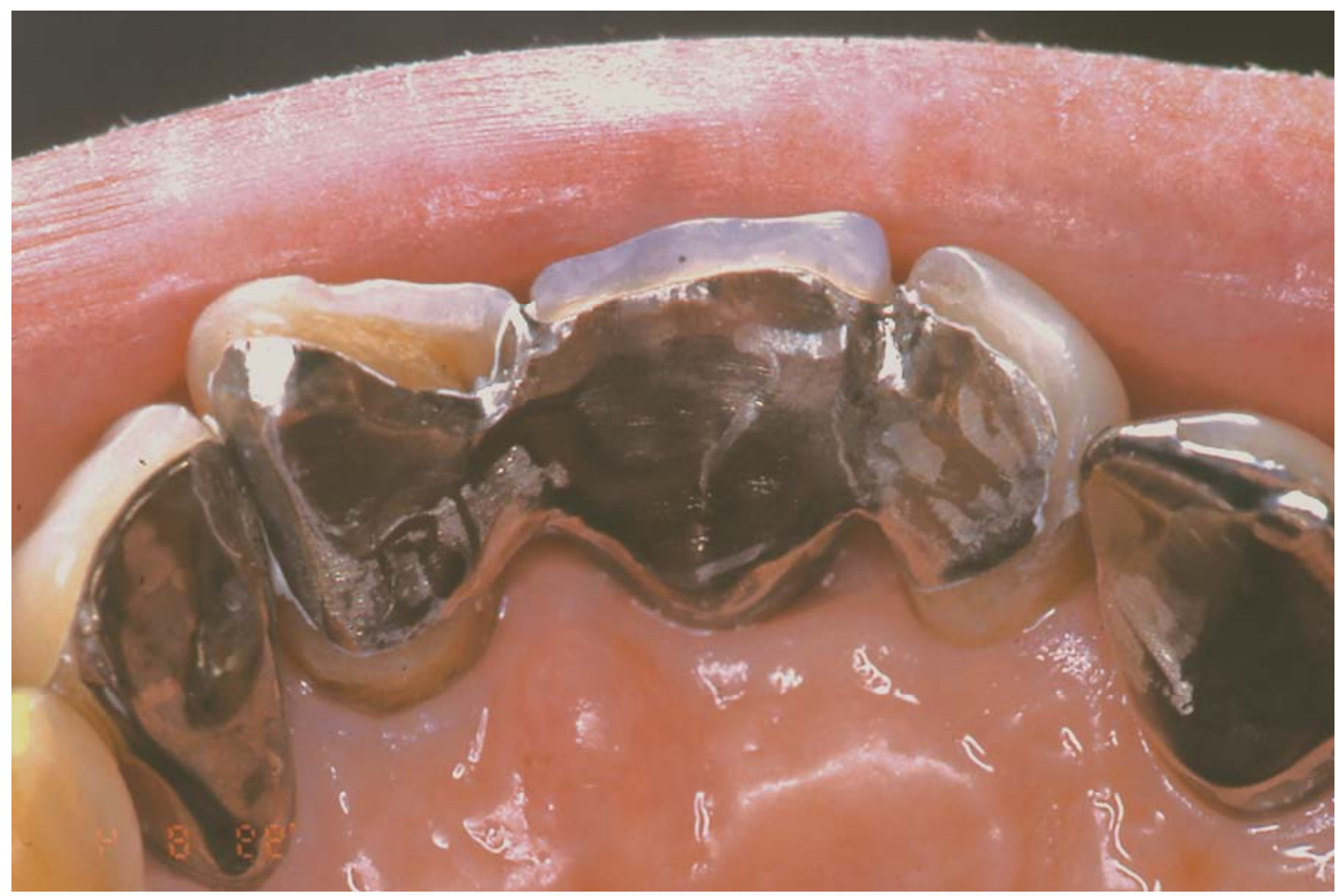


Fig. 3

(A)

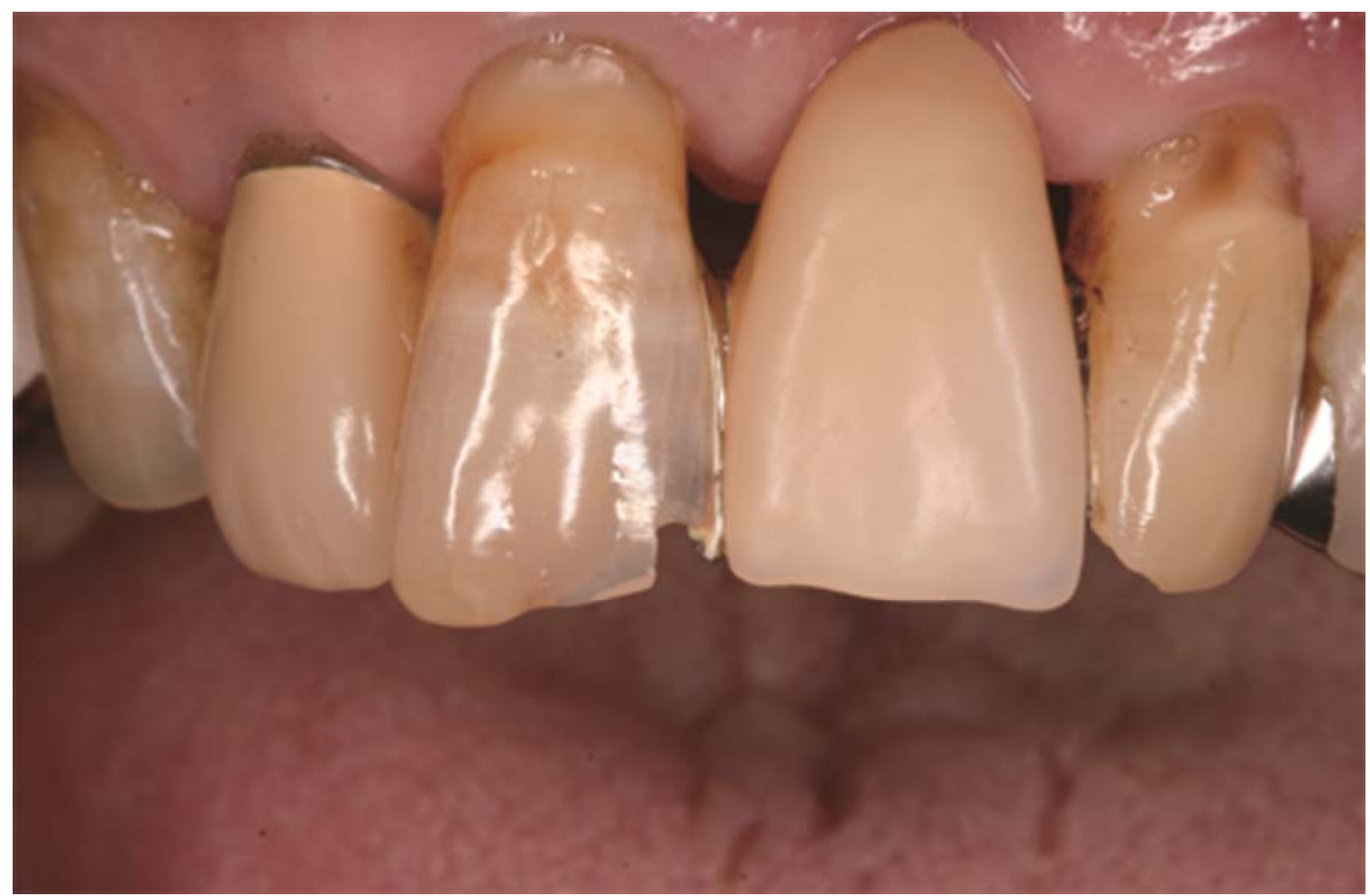


Fig. 3

(B)

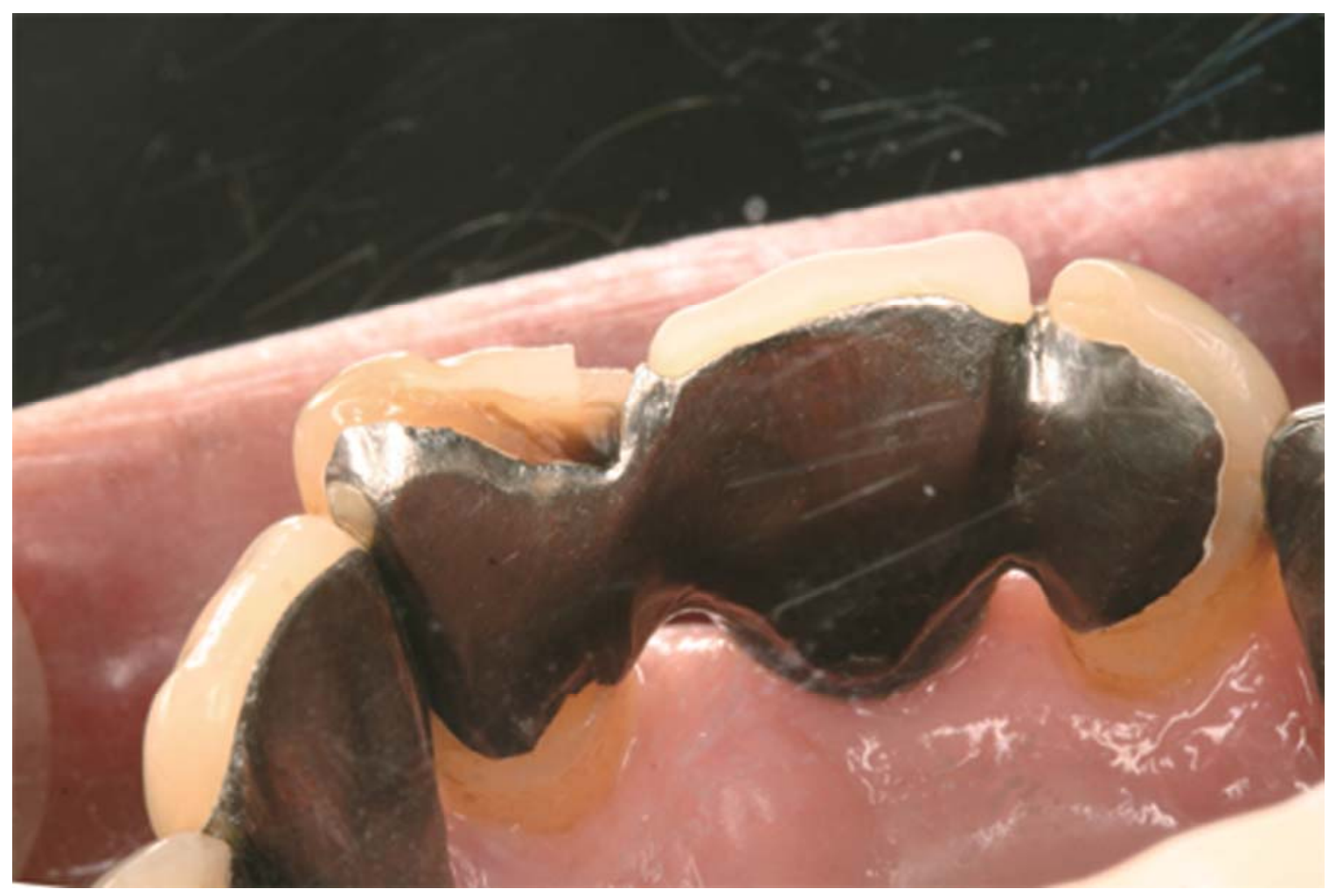


Fig. 4

(A)

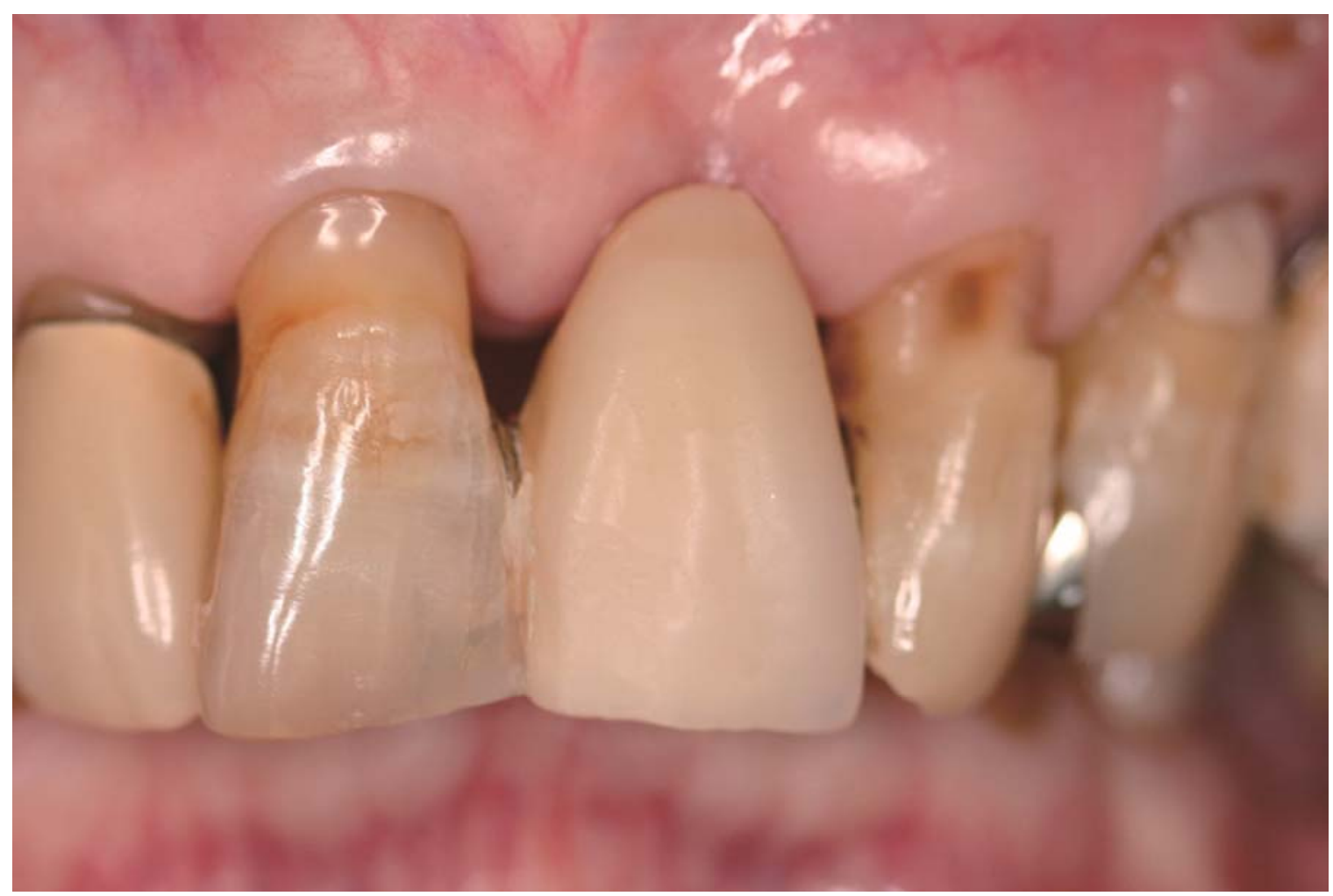


Fig. 4

(B)

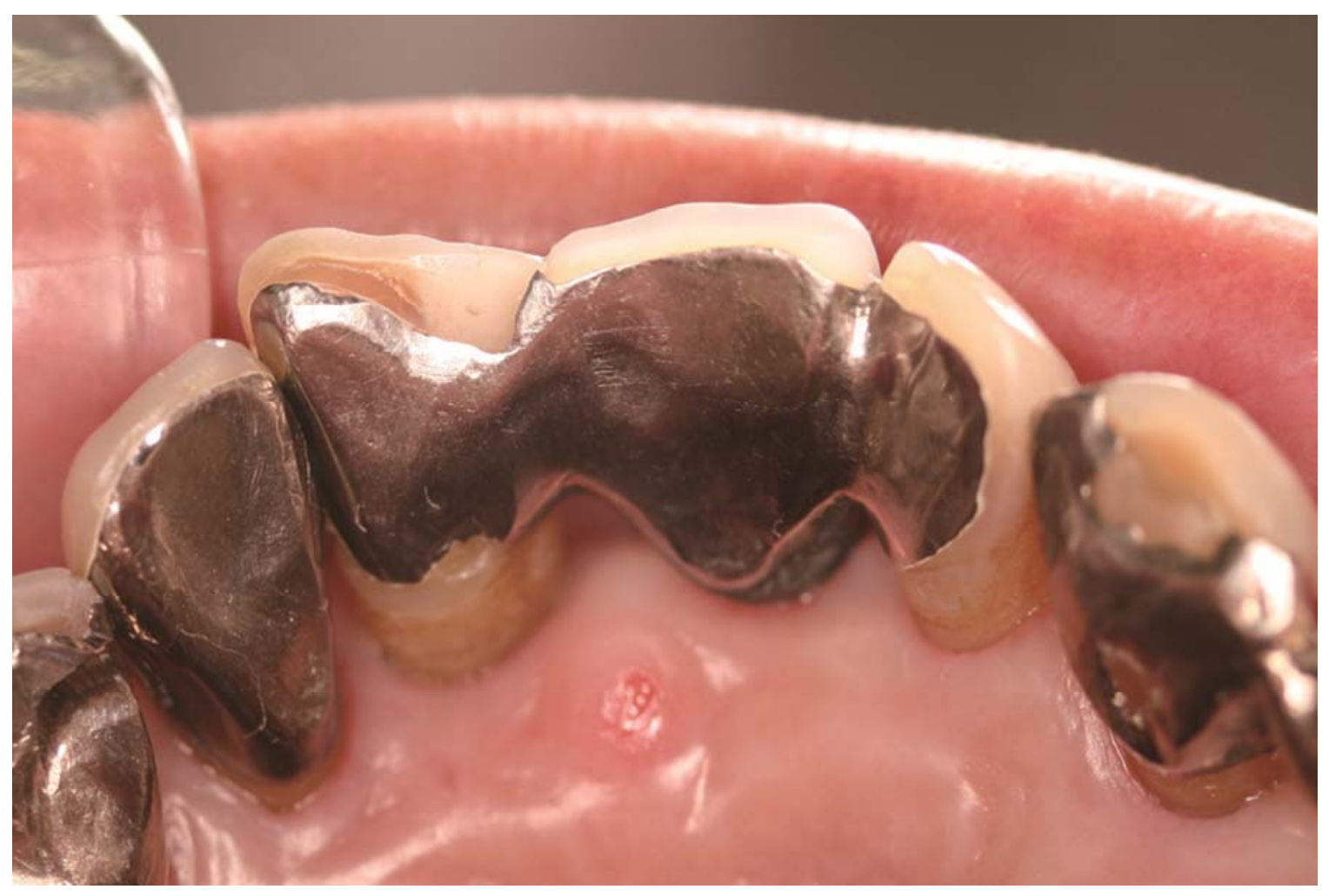

